\title{
Biomarkers of Organophosphate Pesticides and Attention-Deficit/ Hyperactivity Disorder in Children: A Case-Control Study
}

\section{Manal Abd El-Salam ${ }^{1}$, Amal A Hegazy ${ }^{2,3 *}$, Marwa Elhady', Gamil Elsayed Ibrahim ${ }^{4}$ and Rania Hussein ${ }^{5}$}

${ }^{1}$ Pediatrics Department, Faculty of Medicine, Al-Azhar University, Cairo, Egypt

${ }^{2}$ Family and Community Medicine Department, Faculty of Medicine, King Abdulaziz University, Jeddah, Saudi Arabia

${ }^{3}$ Community and Occupational Medicine Department, Faculty of Medicine, Al-Azhar University, Cairo, Egypt

${ }^{4}$ Flavour and Aroma Chemistry Department, National Research Center, Cairo, Egypt

${ }^{5}$ Neuropsychiatric Department, Faculty of Medicine (for girls), Al-Azhar University, Cairo, Egypt

\begin{abstract}
Children are frequently exposed to significant hazard for organophosphate poisoning (OP) in their environment; since the brain is growing during this period and it is extra liable to neurotoxicants. The aim of this work was to determine the relationship between urinary concentrations of dialkylphosphates (DAP) metabolites, which are biomarkers of OP pesticide exposure, and attention-deficit/hyperactivity disorder (ADHD) in Egyptian children ages seven to twelve years. A case-control study was conducted at Alzhraa University hospital. We recruited 40 children diagnosed as ADHD according to "the diagnostic and statistical manual of mental disorder (DSM-5) criteria" by psychiatrist. Control group of 40 healthy children who did not have any psychiatric or neurologic disorders, age and sex matched. Exposure was determined by analyzing six urinary metabolites of DAP. We investigated the association between ADHD subtypes, coincidence of epilepsy and OP exposure. Significant increase in urinary level of all DAP metabolites for children with ADHD in comparison to control group except Diethyl-dithio-phosphate. ADHD children with epilepsy had statistically significant elevated level of urinary Dimethyl-dithio-phosphate, Diethyl- phosphate and Dimethyl-phosphate metabolites in comparison to those without epilepsy. By logistic regression analysis, Children with high level of urinary Dimethyldithio-phosphate $(\mathrm{OR}=2.29)$, Diethyl-phosphate $(\mathrm{OR}=2.40)$, Dimethyl-phosphate $(\mathrm{OR}=2.02)$, and Dimethyl-thiophosphate $(\mathrm{OR}=1.82)$ have two fold increased risk of developing ADHD than those having lower concentration of these metabolites. Our findings back up the theory that existing environmental concentration of organophosphate pesticide exposure may lead to the development of ADHD and increase the risk of epilepsy in ADHD children.
\end{abstract}

Keywords: Organophosphate; Pesticides; ADHD; Dialkylphosphates; DAP; Epilepsy

\section{Introduction}

Farming and occupant use of organophosphate (OP) pesticides has increased than before in recent decades after forbidding some persistent pesticides. Even though there is confirmation of the results of OP on neurodevelopment and behaviour in adults, however, the data about their outcome in children is resticted [1]. Both Farming and inhabitant settings used a lot of million pounds of OP in 2001. The Environmental Protection Agency (EPA) anticipates nutrition, drinking water, and inhabitant pesticide apply as vital sources of exposure [2]. Inhabitant pesticide use is common, but the major origin of exposure to pesticides for infants and children would be the meal, according to the National Academy of Sciences [3].

Taken into consideration children are frequently exposed to significant hazard for organophosphate poisoning; since the brain is growing during this period and it is extra liable to neurotoxicants [4]. Regarding the body weight of children and the amount of pesticides tend to be high. Young children whose age range from six to eleven years having greatest urinary levels of dialkyl-phosphate metabolites (biomarkers of organophosphate exposure), contrast to another group of different age [5]. Body of young children containing minor levels of metabolizing enzymes (paraoxonase or chlorpyrifosoxonase) that disrupt OP pesticides than adults [6,7], indicating that children may be higher susceptible to risk of exposure.

Dialkyl-phosphates (DAP) are metabolites resulting from the degradation of various OP pesticides by the act of esterases. The degradation of most organophosphate pesticides creates only six alkyl-phosphate metabolites which point to that a single investigative technique is adequate to detect exposure to different substances [8]. The six DAP metabolites were three "dimethyl-alkyl-phosphate (DMAP), including dimethyl-phosphate (DMP), dimethyl-thio-phosphate (DMTP), and dimethyl-dithio-phosphate (DMDTP), and three diethylalkyl-phosphate (DEAP) molecules, including diethyl-phosphate (DEP), diethyl-thio-phosphate (DETP), and diethyl-dithio-phosphate (DEDTP) [9].

Numerous biological techniques might cause connection between ADHD and OP pesticides. The main action of these toxic substances, mostly due to acute toxicity, is suppression of acetylcholin esterase [10], and disturbance in cholinergic signals [11]. When the dose lower than that causing acetylcholinesterase suppression, specific OP influence various neurochemical targets, numerous neurotransmitter and second messenger systems, including growth factors $[12,13]$. OP pesticides exposure during growing might have constant effects on multiple neural systems that lead to ADHD behaviors, such as inattention and cognitive deficits, which is similar to the symptoms due to nicotine exposure during development $[14,15]$. Globally ADHD is considered one of the main prevalent neurobehavioral disorders in children, affecting three to ten percent of children [16-18].

${ }^{*}$ Corresponding author: Amal A Hegazy, Family and Community Medicine Department, Faculty of Medicine, King Abdulaziz University, Jeddah, Saudi Arabia Tel: +201063635064; E-mail: renalahmed@gmail.com

Received April 19, 2017; Accepted April 24, 2017; Published April 28, 2017

Citation: El-Salam MA, Hegazy AA, Elhady M, Ibrahim GE, Hussein R (2017) Biomarkers of Organophosphate Pesticides and Attention-Deficit/Hyperactivity Disorder in Children: A Case-Control Study. J Environ Anal Toxicol 7: 460. doi: 10.4172/2161-0525.1000460

Copyright: ( 2017 El-Salam MA, et al. This is an open-access article distributed under the terms of the Creative Commons Attribution License, which permits unrestricted use, distribution, and reproduction in any medium, provided the original author and source are credited. 
Citation: El-Salam MA, Hegazy AA, Elhady M, Ibrahim GE, Hussein R (2017) Biomarkers of Organophosphate Pesticides and Attention-Deficit/ Hyperactivity Disorder in Children: A Case-Control Study. J Environ Anal Toxicol 7: 460. doi: 10.4172/2161-0525.1000460

ADHD manifestations mostly continue into youth and adult, and are linked to considerable social and medical costs $[19,20]$. A lot of studies pointed to that neurodevelopmental troubles, encompassing ADHD, outcome from combined connections of hereditary and ecological causes. There is increasing evidence regarding the role of different toxicants in neurodevelopmental disorders, including ADHD. The growing brain is completely susceptible to substances which are toxic to neurons at low level that does not cause serious effects on adulthood. Even though many successful hard work from public health activities and regulatory agenda restricting the level of exposure to industrial chemicals, although alarms are present as result of their effect, yet at little concentration, on the health of children and development of neurons [21]. Our objective is to determine the association between concentrations of dialkyl-phosphate metabolites in urine, which are biomarkers of organophosphate pesticides exposure in Egyptian children ages seven to twelve years and attention-deficit/hyperactivity disorder.

\section{Materials and Methods}

This case control study was conducted at Alzhraa hospital, ALAzhar University, Cairo, Egypt, between July 2015 and May 2016. It comprised 40 children diagnosed as ADHD according to the diagnostic and statistical manual of mental disorder (DSM-5) criteria [18] by psychiatrist. They were recruited from psychiatric outpatient clinic at Alzahraa University hospital. Another group of 40 healthy children was selected randomly at the same period from outpatient pediatric clinic at Alzahraa University hospital and did not have any psychiatric or neurologic disorders, age and sex matched children served as a control group. Their age ranged between 7 to 12 years old. Informed written parental consent was obtained prior to enrollment in the study in adherence with the guidelines of the ethical committee of Alzhraa hospital, AL-Azhar University, Cairo, Egypt.

Children with any systemic medical illness (e.g., cardiac, respiratory, hepatic, renal diseases, neuromuscular disabilities) or any psychological disorders other than ADHD and parental psychosocial factors were excluded from the study.

\section{Clinical history and examination}

All the studied children were subjected to clinical examination and interview with their parents to fill the questionnaire. Itincluded demographic data, presenting symptoms, any medical disorders, presence of seizures, current medications and history of exposure to passive smoking, chemical pollutant, household or environmental pesticides.

\section{Diagnosis of ADHD}

Children were diagnosed as ADHD according to the DSM- 5 criteria [18] by psychiatrist and children were categorized into (subtypes): hyperactive, inattention or mixed ADHD. Additionally, Conner test was done to all the included children. This test is a diagnostic tool used to screen for ADHD in children and adolescents. The test is used to assess the behaviour, emotion and academic problem and it includes the DSM-5 criteria for diagnosis of ADHD. In this study we used the parent form of Conner test. It is a set of rating scales consist of 43 items with score 0 -3 for each It offers straightforward administration and scoring with excellent validity and reliability. Scores above 60 are usually a sign for having ADHD. Scores between 61 and 70 are usually a sign that the child may have a moderately severe problem. Score more than 70 indicates a more severe problem. Completion of the test takes from 6090 minutes.

\section{Electroencephalogram (EEG)}

Digital interictal EEG was performed using a Nihon Kohden 1200 digital EEG instrument. Intermittent photic stimulation activation procedure was done for all cases. Chloral hydrate sedation was infrequently used in unhelpful children ( $30 \mathrm{mg} / \mathrm{kg}$ maximum $1 \mathrm{gm} / \mathrm{dose})$ that were given 30 minutes before recording with complete monitoring of the vital data during the recording. Regarding history of seizures and EEG results we divided ADHD group into two groups, the first one is ADHD with epilepsy and the other is ADHD without epilepsy.

\section{Laboratory investigation}

Urine collection and urinalysis: Samples of urine were collected, transported on ice to the laboratory without delay, and kept back frozen at $\left(-20^{\circ} \mathrm{C}\right)$ at the time of examination. Before analyzing, the urine samples were liquefied and homogenized using a vortex mixer. Six DAP as biomarkers of organophosphates exposure were analyzed by gas chromatography (GC): DMP, DEP, DMTP, DETP, DMDTP and DEDTP.

Samples of urine were prepared for GC examination according to a modified method of Moate et al. [22]. "Aliquots of the samples underwent azeotropic distillation with methanol and evaporation under a nitrogen stream. Sample extracts were then derivatized with 2,3,4,5,6-pentafluorobenzylbromide to convert phosphate acids to esters. Extracted samples were analyzed on a gas chromatograph (Agilent 7890A GC system equipped with an OI 5380 pulsed flame photometric detector (PFPD)". Creatinine concentrations were measured using the Jaffe reaction [23].

Each metabolite was converted from its untransformed concentration microgram/liter $(\mu \mathrm{g} / \mathrm{L})$ to the corresponding molar concentration $(\mathrm{nmol} / \mathrm{L})$ by dividing by its molecular weight. The concentrations of urinary DEP metabolites were expressed as $\mu \mathrm{g}$ per gram $(\mu \mathrm{g} / \mathrm{gm})$ creatinine.

Statistical analysis: Statistical analysis was performed using the Statistical Package for Social Sciences (version 20; SPSS Inc., Chicago, IL, USA). Quantitative data were expressed as mean \pm SD. Differences between two groups were analyzed with independent $\mathrm{T}$ student test. Differences between more than two groups were analyzed with ANOVA test. Logistic regression analysis was done to determine the predictors of ADHD in the studied children. Receiver operating characteristic curves (ROC) were used to identify the optimal cut-off points of urinary metabolites levels for prediction of ADHD. P-value $<0.05$ was considered to be significant.

\section{Results}

This study carried out at forty children with ADHD (60\% male and $40 \%$ female); twenty (50\%) of them were hyperactive, ten $(25 \%)$ were inattention and ten $(25 \%)$ were mixed type ADHD. The age of onset ranged between two to five years with a mean duration of illness of $6.25 \pm 3.55$ years. Twenty seven of them $(67.5 \%)$ received medication. Twenty two of them (55\%) had EEG abnormalities but only $18(45 \%)$ of them had confirmed diagnosis of epilepsy. Another 40 healthy children were selected as controls ( $62.5 \%$ male and $37.5 \%$ female). There was no significant difference between cases and control regarding age, gender, socioeconomic status, passive smoking, weight and height $(\mathrm{p}=0.632$, $\mathrm{p}=0.053, \mathrm{p}=0.622, \mathrm{p}=0.820, \mathrm{p}=0.528$, and $\mathrm{p}=0.871$, respectively). As demonstrated in Table 1, there was statistically significant increase in urinary level of all DAP metabolites for children with ADHD than control group ( $\mathrm{p}=0.000)$ except DEDTP $(\mathrm{p}=0.948)$.

We observed significant association between ADHD subtypes 
Citation: El-Salam MA, Hegazy AA, Elhady M, Ibrahim GE, Hussein R (2017) Biomarkers of Organophosphate Pesticides and Attention-Deficit/ Hyperactivity Disorder in Children: A Case-Control Study. J Environ Anal Toxicol 7: 460. doi: 10.4172/2161-0525.1000460

(hyperactive, inattention or mixed) regarding urinary level of DEDTP, DMTP and DMP $(\mathrm{p}=0.002, \mathrm{p}=0.001$ and $\mathrm{p}=0.001$, respectively); however there was no significant difference in the rest of dialkylphosphates metabolites [DMDTP $(p=0.469)$, DETP $(p=0.183)$ and DEP $(p=0.389]$. In addition, by Post hoc: LSD test we found significant increase in urinary metabolite of DMP and DME in children with hyperactive versus inattention and mixed type while there was no significant difference between inattention versus mixed type (Table2).

Furthermore ADHD children with epilepsy had statistically significant elevated level of urinary DMDTP $(\mathrm{p}=0.030)$, DEP $(\mathrm{p}=$ $0.007)$ and DMP $(p=0.002)$ metabolites in comparison to those without epilepsy as shown in Table 3. Logistic regression analysis for ADHD predictors of DAP metabolites demonstrated in Table 4. The common predictors of urinary DAP metabolites associated with ADHD were DMDTP $(\mathrm{OR}=2.29)$, DEP $(\mathrm{OR}=2.40)$, DMP $(\mathrm{OR}=2.02)$ and DMTP $(\mathrm{OR}=1.82)$. Children with high level of urinary DMDTP, DEP, DMP, and DMTP were have two fold increased risk of developing ADHD than those having lower concentration of these metabolites.

The cut off points for dialkylphosphates metabolites for ADHD cases were revealed in Table 5; it revealed that DETP is $100 \%$ sensitive and $65 \%$ specific, DMTP is $95 \%$ sensitive and 55\% specific and DMDTP is $80 \%$ sensitive and $90 \%$ specific in prediction of ADHD. However in
ROC curve (Figure 1) shows that the most sensitive predictor tests of ADHD are DEP followed by DMDTP and DETP.

\section{Discussion}

OP pesticides exposure was widespread in our children, with hundred percent of them having DAP metabolites detected in their urine. Our results suggest a connection between childhood urinary dialkyl-phosphate metabolite concentrations, which are biological biomarker of organophosphate pesticides exposure, and ADHD particularly hyperactive-impulsive type. These findings are consistent with previous studies that reported increased ADHD behavioral problems in school-age children with higher organophosphate metabolite concentrations in urine. Lizardi et al. [24] reported adverse link of child urinary DAP and attention related performance errors in children at seven years of age. In this research the children with high level of urinary DMDTP, DEP, DMP, and DMTP were have two fold increased risk of developing ADHD than those having lower concentration of these metabolites. Yu et al. [9] found that higher concentration of DMP in children urine may have a double to triple increased risk of being diagnosed with ADHD. Furthermore, Bouchard et al. [2] reported that Children with elevated levels of dialkyl-phosphate in urine, particularly dimethyl-alkyl-phosphate (DMAP) were more likely to be diagnosed as having ADHD.

\begin{tabular}{|c|c|c|c|c|c|}
\hline & & \multirow{2}{*}{ Control $(n=40)$} & \multirow{2}{*}{$\operatorname{ADHD}(n=40)$} & \multicolumn{2}{|c|}{ test } \\
\hline & & & & t-test & p-value \\
\hline DEDTP (nmol/L) & Mean \pm SD Range & $38.60 \pm 8.3517-51$ & $38.47 \pm 8.8924-53$ & 0.065 & 0.948 \\
\hline DMDTP (nmol/L) & Mean \pm SD Range & $43.55 \pm 9.0628-68$ & $75.95 \pm 14.3145-98$ & 12.095 & $0.000^{*}$ \\
\hline $\operatorname{DETP}(\mathrm{nmol} / \mathrm{L})$ & Mean \pm SD Range & $90.82 \pm 11.3176-114$ & $117.80 \pm 38.0375-184$ & 4.300 & $0.000^{*}$ \\
\hline DMTP (nmol/L) & Mean \pm SD Range & $115.58 \pm 24.0294-183$ & $191.88 \pm 52.07128-312$ & 8.416 & $0.000^{*}$ \\
\hline DEP ( $\mu \mathrm{g} /$ gmcreatinine) & Mean \pm SD Range & $60.50 \pm 14.2146-77$ & $88.32 \pm 15.4267-125$ & 8.390 & $0.000^{*}$ \\
\hline $\mathrm{DMP}(\mathrm{nmol} / \mathrm{L})$ & Mean \pm SD Range & $195.82 \pm 25.46178-247$ & $292.22 \pm 68.64178-451$ & 8.327 & $0.000^{*}$ \\
\hline
\end{tabular}

DEDTP: Diethyl-dithio-phosphate; DMDTP: Dimethyl-dithio-phosphate; DETP: Diethyl-thio-phosphate; DMTP: Dimethyl-thio-phosphate; DEP: Diethyl-phosphate; DMP: Dimethyl-phosphate; *Significant.

Table 1: Comparison between cases and control group regarding level of dialkylphosphates metabolites.

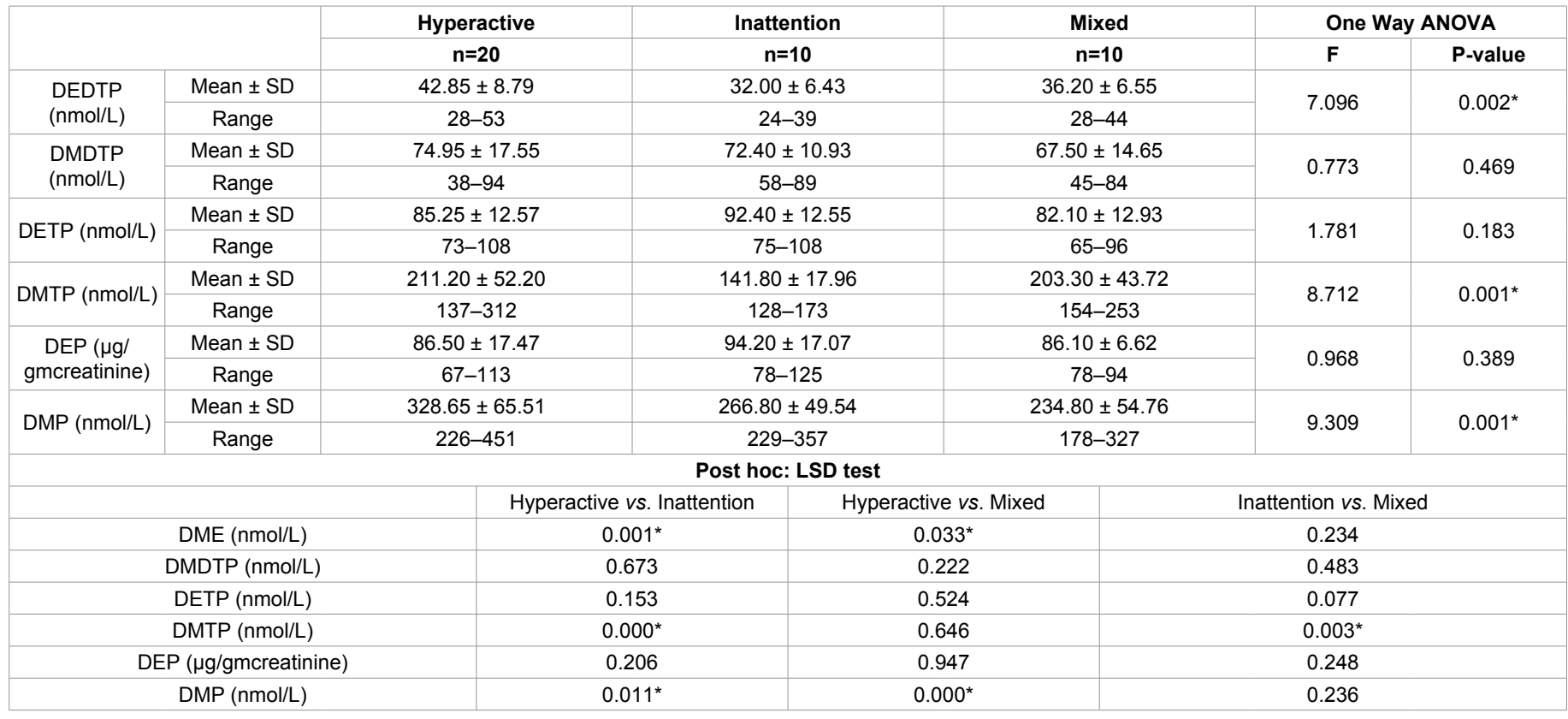

*Significant

Table 2: Level of dialkyl-phosphates metabolites regarding ADHD subtypes. 
Citation: El-Salam MA, Hegazy AA, Elhady M, Ibrahim GE, Hussein R (2017) Biomarkers of Organophosphate Pesticides and Attention-Deficit/ Hyperactivity Disorder in Children: A Case-Control Study. J Environ Anal Toxicol 7: 460. doi: 10.4172/2161-0525.1000460

Page 4 of 5

\begin{tabular}{|c|c|c|c|c|c|}
\hline & & \multirow{2}{*}{$\begin{array}{l}\text { ADHD without epilepsy } \\
\qquad n=22\end{array}$} & \multirow{2}{*}{$\begin{array}{l}\text { ADHD with epilepsy } \\
\qquad n=18\end{array}$} & \multicolumn{2}{|c|}{ Independent t-test } \\
\hline & & & & $\mathbf{t}$ & p-value \\
\hline \multirow{2}{*}{ DEDTP (nmol/L) } & Mean \pm SD & $36.23 \pm 7.15$ & $41.22 \pm 10.17$ & \multirow{2}{*}{1.820} & \multirow{2}{*}{0.077} \\
\hline & Range & $24-47$ & $24-53$ & & \\
\hline \multirow{2}{*}{ DMDTP (nmol/L) } & Mean \pm SD & $67.73 \pm 14.43$ & $78.22 \pm 14.89$ & \multirow{2}{*}{2.256} & \multirow{2}{*}{$0.030^{\star}$} \\
\hline & Range & $38-89$ & $45-94$ & & \\
\hline \multirow{2}{*}{ DETP (nmol/L) } & Mean \pm SD & $82.86 \pm 11.23$ & $90.39 \pm 13.91$ & \multirow{2}{*}{1.894} & \multirow{2}{*}{0.066} \\
\hline & Range & $65-108$ & $73-108$ & & \\
\hline \multirow{2}{*}{ DMTP (nmol/L) } & Mean \pm SD & $172.36 \pm 38.88$ & $215.72 \pm 57.07$ & \multirow{2}{*}{2.849} & \multirow{2}{*}{$0.007^{*}$} \\
\hline & Range & $129-253$ & $128-312$ & & \\
\hline \multirow{2}{*}{ DEP ( $\mu$ g/gmcreatinine) } & Mean \pm SD & $88.77 \pm 15.30$ & $87.78 \pm 16.00$ & \multirow{2}{*}{0.200} & \multirow{2}{*}{0.842} \\
\hline & Range & $68-125$ & $67-113$ & & \\
\hline \multirow{2}{*}{$\mathrm{DMP}(\mathrm{nmol} / \mathrm{L})$} & Mean \pm SD & $260.18 \pm 57.10$ & $325.83 \pm 70.80$ & \multirow{2}{*}{3.248} & \multirow{2}{*}{$0.002^{*}$} \\
\hline & Range & $178-357$ & $231-451$ & & \\
\hline
\end{tabular}

*significant $(p<0.05)$

Table 3: Level of dialkylphosphates metabolites in ADHD children with and without epilepsy.

\begin{tabular}{|c|c|c|c|c|c|c|c|}
\hline & \multirow{2}{*}{ B } & \multirow{2}{*}{ S.E. } & \multirow{2}{*}{ Wald } & \multirow{2}{*}{ P-value } & \multirow{2}{*}{ Odds ratio (OR) } & \multicolumn{2}{|c|}{$95 \% \mathrm{Cl}$} \\
\hline & & & & & & Lower & Upper \\
\hline DMDTP & 0.117 & 0.029 & 23.15 & 0.000 & 2.29 & 1.06 & 2.02 \\
\hline DETP & -0.108 & 0.030 & 12.45 & 0.000 & 0.89 & 0.84 & 0.95 \\
\hline DMTP & 0.044 & 0.008 & 15.75 & 0.000 & 1.82 & 1.30 & 2.03 \\
\hline DEP & 0.225 & 0.078 & 25.04 & 0.000 & 2.40 & 1.68 & 2.72 \\
\hline DMP & 0.141 & 0.017 & 18.77 & 0.000 & 2.02 & 1.21 & 2.23 \\
\hline
\end{tabular}

Table 4: Logistic regression analysis for the predictors of ADHD in the studied children.

\begin{tabular}{|c|c|c|c|c|c|c|}
\hline & Cut off point & AUC & Sensitivity & Specificity & $+P V$ & -PV \\
\hline DMDTP & $>61$ & 0.904 & 80 & 90 & 94.1 & 69.2 \\
\hline DETP & $\leq 108$ & 0.91 & 100 & 65 & 85.1 & 100 \\
\hline DMTP & $>128$ & 0.779 & 95 & 55 & 80.9 & 84.6 \\
\hline DEP & $>77$ & 0.937 & 77.5 & 95 & 96.9 & 67.9 \\
\hline DMP & $>238$ & 0.789 & 70 & 85 & 90.3 & 58.6 \\
\hline
\end{tabular}

Table 5: Cut off point for the level of dialkyl-phosphate metabolites among ADHD cases.

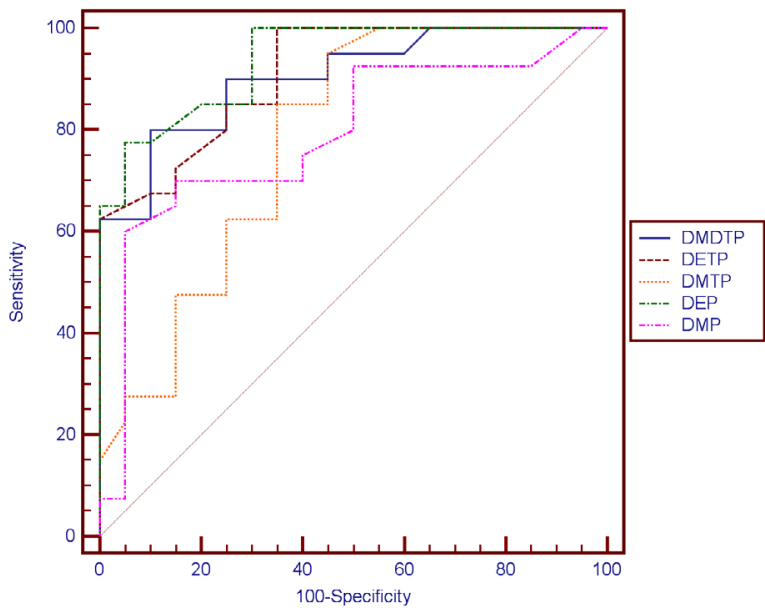

Figure 1: ROC curve for urinary level of dialkylphosphates metabolites in ADHD cases.

A study was done at United State observed that more than ninety percent of children have noticeable urinary residues of DAP [25]. The half-life of DAP metabolites range from twenty four to twenty seven hours and three to six days for complete removal from the body, the recognition of high rate of DAP point to that children are constantly exposed to OP pesticides [26]. Children may get bigger exposure than adults since they eat, drink, and respire more per unit of body weight. In addition, children are further critical to the effects of toxic substances as their bodies have lower detoxification abilities [27].

The associations between organophosphate pesticides and ADHD in children may be explained by several biological mechanisms. A main mechanism of organophosphates, mainly with regard to acute poisoning, is suppression of acetylcholinesterase [28] and disturbances in cholinergic signaling are consider taking place in ADHD [29]. At dosages minor than those required to supress acetylcholinesterase, certain organophosphates affect different neurochemical targets, including disruption of the nuclear transcription factors, interference with neural cell development, alter the synaptic formation [30], reduced muscarinic and nicotinic acetylcholine receptor expression, DNA synthesis inhibition, generation of reactive oxygen species [31], dysregulation of neuronal signal pathways and has neuronal excitatory effects [32]. These effects occur at lower organophosphate concentrations that did not inhibit acetylcholinesterase so cholinergic transmission remains unaffected [33].

Our results revealed that elevated urinary organophosphate metabolite levels are associated with increase the coincidence of epilepsy in ADHD children. Verhulst et al. [33] reported that thirty percent of children intoxicated with OP developed tonic-clonic- 
Citation: El-Salam MA, Hegazy AA, Elhady M, Ibrahim GE, Hussein R (2017) Biomarkers of Organophosphate Pesticides and Attention-Deficit/ Hyperactivity Disorder in Children: A Case-Control Study. J Environ Anal Toxicol 7: 460. doi: 10.4172/2161-0525.1000460

Page 5 of 5

convulsions. Seizures are more frequent in children after exposure to OP pesticide poisoning than in adults [34]. Exposure to a neurotoxic compound during a vital period of growth may only effect later in life because exposure takes place at a time when sequencing growth processes happen [35,36].

\section{Conclusion and Recommendation}

Our children continuously exposed to organophosphate pesticides in their environment. The present study support the building up information that connecting elevated concentrations of pesticide exposure to unfavorable neuro-developmental results. Results of this study back up the theory that existing environmental concentration of organophosphate pesticide exposure may lead to the development of ADHD and increase the risk of epilepsy in ADHD children. In view of these findings, we recommend the implementation of measures in the form of policies and regulations to control the use of pesticides in the farm and homes. Additionally, increase the public awareness and health education of the general population about the hazard of pesticides and how to avoid the exposure especially in children.

\section{References}

1. González-Alzaga B, Lacasaña M, Aguilar-Garduño C, Rodríguez-Barranco M, Ballester F, et al. (2013) A systematic review of neurodevelopmental effects of prenatal and postnatal organophosphate pesticide exposure. Toxicol Lett 230: 104-121.

2. Bouchard MF, Bellinger DC, Wright RO, Weisskopf MG (2010) AttentionDeficit/Hyperactivity Disorder and Urinary Metabolites of Organophosphate Pesticides. Pediatrics 125: e1270-1277.

3. US National Research Council, Committee on Pesticides in the Diets of Infants and Children.

4. Pesticides in the Diets of Infants and Children (1993) National Academy Press, Washington, DC.

5. Weiss $B(2000)$ Vulnerability of children and the developing brain to neurotoxic hazards. Environ Health Perspect 108 Suppl 3: 375-381.

6. Barr DB, Bravo R, Weerasekera G, Caltabiano LM, Whitehead RD Jr, et al. (2004) Concentrations of dialkyl phosphate metabolites of organophosphorus pesticides in the U.S. population. Environ Health Perspect 112: 186-200.

7. Furlong CE, Holland N, Richter RJ, Bradman A, Ho A, et al. (2006) PON1 status of farmworker mothers and children as a predictor of organophosphate sensitivity. Pharmacogenet Genomics 16: 183-190.

8. Holland N, Furlong C, Bastaki M, Richter R, Bradman A, et al. (2006) Paraoxonase polymorphisms, haplotypes, and enzyme activity in Latino mothers and newborns. Environ Health Perspect 114: 985-991.

9. Bravo R, Caltabiano LM, Weerasekera G, Whitehead RD, Fernandez C, et al. (2004). Measurement of dialkyl phosphate metabolites of organophosphorus pesticides in human urine using lyophilization with gas chromatographytandem mass spectrometry and isotope dilution quantification. J Expo Sci Env Epid 14: 249-259.

10. Yu CJ, Du JC, Chiou HC, Chung MY, Yang W, et al. (2016) Increased risk of attention-deficit/hyperactivity disorder associated with exposure to organophosphate pesticide in Taiwanese children. Andrology 4: 695-705.

11. Sultatos LG (1994) Mammalian toxicology of organophosphorus pesticides. J Toxicol Environ Health 43: 271-289.

12. Coccini T, Crevani A, Rossi G, Assandri F, Balottin U, et al. (2009) Reduced platelet monoamine oxidase type $B$ activ-ity and lymphocyte muscarinic receptor binding in unmedicatedchildren with attention deficit hyperactivity disorder. Biomarkers 14: 513-522.

13. Verma SK, Kumar V, Gill KD (2009) An acetylcholinesterase-independent mechanism for neurobehavioral impairments after chronic low level exposure to dichlorvos in rats. Retracted Article See: Retraction Notice.

14. Slotkin TA, Seidler FJ (2007) Comparative developmental neurotoxicity of organophosphates in vivo: transcriptional responses of pathways for brain cell development, cell signaling, cytotoxicity and neurotransmitter systems. Brain Res Bull 72: 232-274.

15. Heath CJ, Picciotto MR (2009) Nicotine-induced plasticity during development: modulation of the cholinergic system and long- term consequences for circuits involved in attention and sensory processing. Neuropharmacology 56: 254-262.

16. Slotkin TA (2004) Cholinergic systems in brain development and disruption by neurotoxicants: nicotine, environmental tobacco smoke, organophosphates. Toxicol Appl Pharmacol 198: 132-151.

17. Polanczyk G, de Lima MS, Horta BL, Biederman J, Rohde LA (2007) The worldwide prevalence of ADHD: a systematic review and metaregression analysis. Am J Psychiatry 164: 942-948.

18. Aguiar A, Eubig PA, Schantz SL (2010) Attention deficit/hyperactivity disorder: a focused overview for children's environmental health researchers. Environ Health Perspect 118: 1646-1653.

19. American Psychiatric Association (2013) Diagnostic and statistical manual of mental disorders ( $5^{\text {th }}$ Edn). Arlington, VA: American Psychiatric Publishing.

20. World Health Organization (1993) The ICD-10 Classification on Mental and Behavioral Disorders: Diagnostic Criteria for Re-search. Geneva: World Health Organization.

21. Smith AK, Mick E, Faraone SV (2009) Advances in genetic studies of attentiondeficit/hyperactivity disorder. Curr Psychiatry Rep 11: 143-148.

22. Polanska K, Jurewicz J, Hanke W (2013) Review of current evidence on the impact of pesticides, polychlorinated biphenyls and selected metals on attention deficit / hyperactivity disorder in children. Int J Occup Med Environ Health 26: 16-38.

23. Moate T, Lu C, Fenske RA, Hahne R, Kalman DA (1999) Improved cleanup and determination of dialkyl phosphates in the urine of children exposed to organophosphorus insecticides. J Anal Toxicol 23: 230-236.

24. Fabiny DL, Ertingshausen G (1971) Automated reaction-rate method for determination of serum creatinine with the CentrifiChem. Clin Chem 17: 696-700.

25. Lizardi PS, O'Rourke MK, Morris RJ (2008) The effects of organophosphate pesticide exposure on Hispanic children's cognitive and behavioral functioning J Pediatr Psychol 33: 91-101.

26. Schettler T (2001) Toxic threats to neurologic development of children. Environ Health Perspect 109 Suppl 6: 813-816.

27. Huen K, Bradman A, Harley K, Yousefi P, Barr DB, et al. (2012) Organophosphate pesticide levels in blood and urine of women and newborns living in an agricultural community. Environ Res 117: 8-16.

28. Costa LG (2006) Current issues in organophosphate toxicology. Clin Chim Acta 366: $1-13$.

29. Sultatos LG (1994) Mammalian toxicology of organophosphorus pesticides. J Toxicol Environ Health 43: 271-289.

30. Coccini T, Crevani A, Rossi G (2009) Reducedplatelet monoamine oxidase type B activity and lymphocyte muscarinic receptor bindingin unmedicated children with attention deficit hyperactivity disorder. Biomarkers 14: 513-522.

31. Flaskos $J$ (2014) The neuronal cytoskeleton as a potential target in the developmental neurotoxicity of organophosphorothionate insecticides. Basic Clin Pharmacol Toxicol 115: 201-208.

32. Torres-Altoro MI, Mathur BN, Drerup JM, Thomas R, Lovinger DM, et al (2011) Organophosphates dysregulate dopamine signaling, glutamatergic neurotransmission, and induce neuronal injury markers in striatum. J Neurochem 119: 303-313.

33. Androutsopoulos VP, Hernandez AF, Liesivuori J, Tsatsakis AM (2013) A mechanistic overview of health associated effects of low levels of organochlorine and organophosphorous pesticides. Toxicology 307: 89-94.

34. Verhulst L, Waggie Z, Hatherill M, Reynolds L, Argent A (2002) Presentation and outcome of severe anticholinesterase insecticide poisoning. Arch Dis Childhood 86: 352-355.

35. Tattersall J (2009) Seizure activity post organophosphate exposure. Front Biosci (Landmark Ed) 14: 3688-3711.

36. Miodovnik A (2011) Environmental neurotoxicants and developing brain. Mt Sinai J Med 78: 58-77. 